Cuadernos de Economía, Vol. 42 (Маyo), pp. 79-101, 2005

\title{
El Tramo Corto de la Estructura a Plazo como Predictor de ExPeCtativas de la ACTIVIDAd ECONÓMICA EN COLOMBIA*
}

\author{
Luis E. ArANGo** \\ LUZ AdRIANA FlóREZ \\ Angélica M. Arosemena \\ Banco de la República, Colombia
}

This article tests the hypothesis that the term structure of interest rates contains information about future economic activity in Colombia. According to the multilogit approach used to verify the hypothesis that an increase in the spread of interest rates reduce the probability of having a rather poor economic performance in the future. This result, in correspondence with the theoretical model, holds for the period between 12 and 24 months ahead. The inclusion of monetary variables in the empirical model affects neither the statistical significance nor the signs of both the spread the inflation differential for the period between 12 and 24 months ahead. However, the growth of monetary base also contains information about the economic environment in the next future.

JEL: E43, E32

Keywords: Estructura a Plazo, Spread de Tasas de Interés, Diferencial de Inflación, Expectativas de Actividad Económica, Logit Ordenados

\section{INTRODUCCIÓN}

La estructura a plazo de las tasas de interés contiene información que ha sido explotada en múltiples formas por los economistas. ${ }^{1}$ Una de sus principales

\footnotetext{
*Las opiniones contenidas en este documento son responsabilidad exclusiva de los autores y no comprometen al Banco de la República ni a sus directivas. Este trabajo fue presentado en las 9as. Jornadas de Economía Monetaria e Internacional, UNLP. Los autores agradecen los comentarios y sugerencias de Cristina Fernández, Luis Fernando Melo, Carlos Esteban Posada, Álvaro Riascos y Leonardo Villar y asumen toda responsabilidad por los errores que pudiera contener el trabajo.

***E-mail: larangth@ banrep.gov.co

${ }^{1}$ Arosemena y Arango (2002) presentan algunos de los usos dados en la literatura a la información derivada de la estructura a plazo de las tasas de interés.
} 
lecturas tiene que ver con los mecanismos de transmisión de la política monetaria hacia las expectativas de inflación y de actividad económica.

Para ganar intuición sobre el contenido informativo de las tasas de interés en materia de actividad económica futura se debe tener en cuenta que los precios de los activos se determinan, por los mercados, mirando hacia adelante. La razón de ello es que, en condiciones normales, dichos precios equivalen al valor presente de la corriente de ingresos futuros que reportaría el activo. Este valor presente se calcula utilizando un factor de descuento construido con base en una tasa de retorno nominal apropiada para cada momento en que se espera recibir ingresos derivados del título que se posee. La tasa de retorno nominal contiene dos elementos conectados a la actividad económica futura: la tasa de interés real y las expectativas del mercado sobre la inflación, término este asociado, además, a la política económica futura.

Por ejemplo, un aplanamiento de la curva de rendimientos estaría prediciendo una caída en las tasas de interés futuras, la cual se podría asociar con un menor ritmo de actividad económica. Si los participantes en el mercado esperan una contracción económica el año siguiente, ellos cambiarán sus inversiones de corto plazo por inversiones de largo plazo para beneficiarse de las ganancias de capital consistentes con la caída en las tasas de interés. Estas decisiones de portafolio significan, respectivamente, que los inversionistas venderán títulos de corto plazo, lo cual llevará a una disminución en su precio o un aumento en las tasas de interés de corto plazo y comprarán títulos de largo plazo, lo cual producirá un aumento de precio o una disminución en las tasas de interés. En consecuencia, el perfil observado en las tasas de interés tendrá una tendencia a la baja tal como se espera por parte de los agentes.

Lo anterior pone de manifiesto que las tasas de interés utilizadas para determinar el precio de los activos contienen información sobre el desempeño económico futuro y que eventualmente es posible extraer total o parcialmente dicha información de las tasas de interés.

Para verificar la hipótesis sobre la capacidad informativa de la curva de rendimientos el trabajo utiliza la ecuación de Euler derivada de un modelo de agente representativo que predice una asociación positiva entre el spread de tasas de interés nominales, definido como la diferencia en el retorno de dos títulos de igual riesgo que tienen vencimientos diferentes, y la actividad económica futura, y una relación negativa entre esta última y el diferencial de inflación. El desempeño de la actividad económica futura se mide con base en el modelo no lineal de Arango y Melo (2005), basado en los métodos de Granger y Teräsvirta (1993) y Teräsvirta (1998).

Este trabajo, el cual es posible llevarlo a cabo gracias al reciente avance del mercado de capitales en Colombia, tiene por objeto explorar la hipótesis de que la curva de rendimientos contiene información acerca del desempeño de la actividad económica futura. Contribuye, en cierta medida, a la corriente de investigación sobre indicadores líderes para Colombia alimentada, entre otros, por Melo et al., Maurer et al. (1996) y Melo et al. (2003). 
El trabajo se desarrolla de la siguiente manera. La sección 2 hace una breve presentación de trabajos realizados para otros países sobre esta línea de investigación. La sección 3 presenta un modelo que destaca el vínculo entre las expectativas de la actividad económica futura y la estructura a plazo de las tasas de interés nominales y el diferencial de inflación. En la sección 4 se describen los datos utilizados para realizar las pruebas empíricas. En la sección 5 se discuten los resultados econométricos. La sección 6 muestra los resultados econométricos de algunos ejercicios empíricos en los que se incluyen variables monetarias al modelo básico de la sección anterior. Finalmente, en la sección 7 hay unas breves conclusiones sobre el trabajo.

\section{Literatura Previa}

La relación entre las tasas de interés y la actividad económica ha sido ampliamente documentada en la literatura. ${ }^{2}$ De igual manera, la asociación entre la estructura a plazo de tasas de interés y la actividad económica ha recibido suficiente atención (Fama, 1986; Hu, 1993). En materia empírica, sin embargo, el éxito de dicha asociación es relativo. Así, por ejemplo, Harvey (1988), utilizando un modelo teórico en el que hace explícita una relación lineal entre la estructura a plazo y el crecimiento futuro del consumo de la economía norteamericana, en términos reales, encuentra que esta última variable se puede pronosticar mejor para los años 70 y 80 que para períodos anteriores.

Para el caso de Canadá, Harvey (1997), utilizando un modelo de similares características, encuentra que la estructura a plazo, derivada del mercado de bonos, contiene información importante sobre la evolución de la actividad real. Los resultados de Estrella y Hardouvelis (1991) sugieren que la pendiente de la curva de rendimientos pronostica el ritmo de actividad económica futura. Dotsey (1998), con las mismas variables de Estrella y Hardouvelis (1991), pero con una muestra mayor en 9 años, concluye que, en Estados Unidos, la pendiente de la curva de rendimientos es una guía útil pero imperfecta de la evolución de la actividad económica. Una definición similar de las variables es empleada por Kim y Limpaphayom (1997) quienes, para el caso de Japón, encuentran que con la estructura a plazo de tasas de interés pueden predecir el crecimiento del producto doméstico durante el período 1984-1991, pero no durante el período 1975-1983.

Bernard y Gerlach (1996) analizan los casos de Bélgica, Canadá, Francia, Alemania, Japón, Países Bajos, Reino Unido y Estados Unidos. Sus estimaciones permiten concluir que, a nivel general, la pendiente de la estructura a plazo es un indicador útil de la actividad económica futura en horizontes de 2, 3 y 4 trimestres. Sin embargo, los resultados sugieren diferencias importantes entre países. Los mejores resultados se obtienen para Alemania, Canadá y Estados Unidos mientras que para Japón el modelo tiene un desempeño menos satisfactorio.

${ }^{2}$ Las referencias se remontan a Fisher (1097) según citas hechas, entre otros, por Harvey (1988) y Kim y Limpaphayom (1997). 
Estrella y Mishkin (1995) muestran que para el corto plazo los indicadores líderes y el PIB rezagado son los mejores predictores de la actividad económica futura a pesar de que su nivel de significancia decrece significativamente en un horizonte de un año. Variables financieras como los índices accionarios y el spread de tasas de interés de los papeles comerciales exhiben un comportamiento similar al de los indicadores líderes mientras que la pendiente de la curva de rendimientos, en todos los horizontes de pronóstico, es capaz de predecir acertadamente la ocurrencia de una recesión económica. De acuerdo con los autores, los agregados monetarios nominales y reales no predicen la actividad económica. Incluyendo diferentes variables explicativas, Estrella y Mishkin (1995) concluyeron que, en la mayoría de los casos, no se mejora la capacidad de pronóstico del modelo, permitiendo así concluir que la pendiente de la curva de rendimientos es el mejor predictor disponible de la actividad económica. Estrella y Mishkin (1996) examinan la capacidad de la curva de rendimientos y otras variables económicas para predecir las recesiones de los Estados Unidos. En este caso, la curva de rendimientos también juega un papel importante.

Finalmente, Bosner-Neal y Morley (1997) encuentran que, dentro de muestra, la pendiente de la curva de rendimientos contiene información relevante para pronosticar la actividad económica futura. Sin embargo, el grado de importancia y la precisión de ésta variable cambian de país a país. Así, por ejemplo, para el caso de Japón, el spread no tiene ninguna capacidad de anticipar cambios futuros en la producción. Para Australia, Países Bajos, Suecia, Suiza y Reino Unido, el spread de tasas de interés puede explicar hasta el $20 \%$ del crecimiento trimestral anualizado del PIB un año adelante, mientras que para Francia, Alemania, Italia y Estados Unidos, la curva de rendimientos explica entre $25 \%$ y $40 \%$ del cambio porcentual del PIB del próximo año.

\section{Estructura a Plazo como Predict or de las Expectativas de Evolución de la Actividad Económica: Guía TeóRICA}

En esta sección se discute la capacidad de la curva de rendimientos para predecir el comportamiento de las expectativas sobre el desempeño económico futuro. Para tal efecto se considera una ecuación de Euler derivada de un modelo de optimización neoclásico de un agente representativo: ${ }^{3}$

$$
I=\beta R_{t} E_{t}\left[\frac{U^{\prime}\left(C_{t+1}\right)}{U^{\prime}\left(C_{t}\right)}\right]
$$

\footnotetext{
${ }^{3}$ Este modelo tiene algunas similitudes con Harvey (1988) en el sentido que se apoya en el consumo como proxy de actividad económica. Sin embargo, veremos más adelante que para contrastar empíricamente nuestra hipótesis utilizamos el índice de producción industrial.
} 
siendo $U^{\prime}\left(C_{t}\right)$ la utilidad marginal del consumo del período $t, R_{t}$ tasa de retorno real bruta de los bonos libres de riesgo, $E_{t}$ el operador de expectativas, ${ }^{4}$ y $\beta$ el factor de descuento, el cual suponemos constante por simplicidad. De acuerdo con (1) las decisiones de los agentes hacen que el consumo varíe hasta el punto en el que la tasa marginal de sustitución esperada del consumo en $t$ y el consumo en $t+1$ iguale la diferencia entre la tasa de impaciencia del agente y la tasa de retorno que ofrece el mercado por diferir el consumo. Dicho de otra manera, dada la tasa subjetiva de descuento, una mayor tasa de interés, posiblemente asociada con una mayor productividad del capital fruto de un choque tecnológico, permite esperar un mayor consumo futuro.

$\mathrm{Si}$ el agente tiene acceso a bonos con vencimiento uno y dos periodos adelante, las ecuaciones de Euler asociadas son:

$$
I=\beta R_{l t} E_{t}\left[\frac{U^{\prime}\left(C_{t+1}\right)}{U^{\prime}\left(C_{t}\right)}\right] \Rightarrow R_{1 t}^{-1}=\beta E_{t}\left[\frac{U^{\prime}\left(C_{t+1}\right)}{U^{\prime}\left(C_{t}\right)}\right]
$$

$\mathrm{y}$,

$$
I=\beta^{2} R_{2 t} E_{t}\left[\frac{U^{\prime}\left(C_{t+2}\right)}{U^{\prime}\left(C_{t}\right)}\right] \Rightarrow R_{2 t}^{-1}=\beta^{2} E_{t}\left[\frac{U^{\prime}\left(C_{t+2}\right)}{U^{\prime}\left(C_{t}\right)}\right]
$$

siendo $R_{1 t}=(1+\eta)$ el retorno bruto de un activo libre de riesgo entre $t$ y $t+1$, $R_{2 t}=\left(1+r_{2}\right)$ el retorno bruto entre $t$ y $t+2$ y $r$ latasa deinterés real. Dividiendo (3) en (2) se obtiene

$$
\frac{R_{2 t}}{R_{1 t}}=\beta E_{t}\left[\frac{U^{\prime}\left(C_{t+1}\right)}{U^{\prime}\left(C_{t}\right)}\right] / \beta^{2} E_{t}\left[\frac{U^{\prime}\left(C_{t+2}\right)}{U^{\prime}\left(C_{t}\right)}\right]
$$

Aplicando logaritmos a (4) y reordenando se obtiene:

$$
\ln E_{t}\left[\frac{U^{\prime}\left(C_{t+1}\right)}{U^{\prime}\left(C_{t}\right)}\right]-\ln E_{t}\left[\frac{U^{\prime}\left(C_{t+2}\right)}{U^{\prime}\left(C_{t}\right)}\right]=\ln \beta+\left(r_{2}-r_{1}\right)
$$

donde la resta del lado izquierdo puede ser mayor, menor o igual que cero dependiendo de las expectativas sobre la evolución de la actividad económica futura. Por ejemplo, partiendo de una situación inicial de equilibrio, donde $C_{t+1}=C_{t}$, se tiene que la tasa marginal de sustitución del consumo en $t$ y $t+1$ será igual a 1 y su

\footnotetext{
${ }^{4}$ Se supone que el agente utiliza toda la información disponible hasta el final del período $t$.
} 
logaritmo igual a cero. Si de este estado de equilibrio inicial la economía pasa a una situación económica favorable tal que $C_{t+2}>C_{t}$ se tendrá una utilidad marginal de consumir en dicho periodo menor que la del periodo corriente:

$$
U^{\prime}\left(C_{t+2}\right)<U^{\prime}\left(C_{t}\right) \Rightarrow \frac{U^{\prime}\left(C_{t+2}\right)}{U^{\prime}\left(C_{t}\right)}<1
$$

y el logaritmo será menor que cero de manera que el lado izquierdo de la expresión se vuelve positivo. Nótese que $\ln E_{t}\left[U^{\prime}\left(C_{t+2}\right) / U^{\prime}\left(C_{t}\right)\right]$ será más negativo cuanto mayor sea la expectativa de auge económico (menor la tasa marginal de sustitución esperada) y, por tanto, la relación entre el spread de tasas reales de interés $\left(r_{2}-r_{1}\right)$ y las expectativas de cambio en el ritmo de actividad económica será positiva.

Quienes están familiarizados con los conceptos de suavización de consumo y sustitución intertemporal saben que la información contenida por las tasas de interés puede generar predicciones diferentes dependiendo del horizonte de tiempo que se considere, como se puede apreciar en el Diagrama 1. Para analizarlo, suponga que se presenta un choque de productividad positivo en el momento $t_{1+\delta}$ que eleva la tasa de interés real de largo plazo $\left(r_{2}\right)$ en relación con la de corto plazo $\left(r_{l}\right)$ y reduce el consumo per cápita en el corto plazo ${ }^{5}$ (entre $t_{1+\delta}$ y $t_{l+2 \delta}$, digamos). ${ }^{6}$ Sin embargo, la actividad económica total $(P I B)$ no muestra ningún impacto significativo dado el aumento que se espera que tenga la inversión al mismo tiempo. ${ }^{7}$ De todas maneras, como lo sugiere la ecuación de Euler, en un futuro cercano la tasa de crecimiento del consumo (y de la actividad económica agregada) aumentará en comparación con el momento $t_{l}$. Al final, el consumo per cápita se ubicará por encima del nivel inicial lo cual indicaría que los agentes juzgarán que un buen ambiente económico futuro es más probable.

Para analizar la relación entre la estructura a plazo de las tasas de interés nominales y la trayectoria futura esperada de la actividad económica podemos utilizar la ecuación de Fisher. De esta manera, queda planteada la existencia de una relación positiva entre la estructura a plazo de las tasas de interés nominal y el crecimiento económico futuro esperado y negativa entre éste último y el diferen-

\footnotetext{
${ }^{5}$ El cambio en el consumo depende de la magnitud de los efectos ingreso y sustitución. El resultado que se presenta aquí supone que domina el efecto sustitución.

${ }^{6}$ Suponemos que $\delta$ es un número positivo pero pequeño.

${ }^{7}$ El efecto neto en el PIB del aumento en la inversión y la caída en el consumo depende del grado de flexibilidad de los precios y del grado de movilidad sectorial de los recursos de la economía.
} 


\section{DIAGRAMA 1. EVOLUCION DEL CONSUMO PER CAPITA DESPUES DE UN AUMENTO DE LA TASA DE INTERES REAL DE LARGO PLAZO EN RELACION CON LA DE CORTO PLAZO}

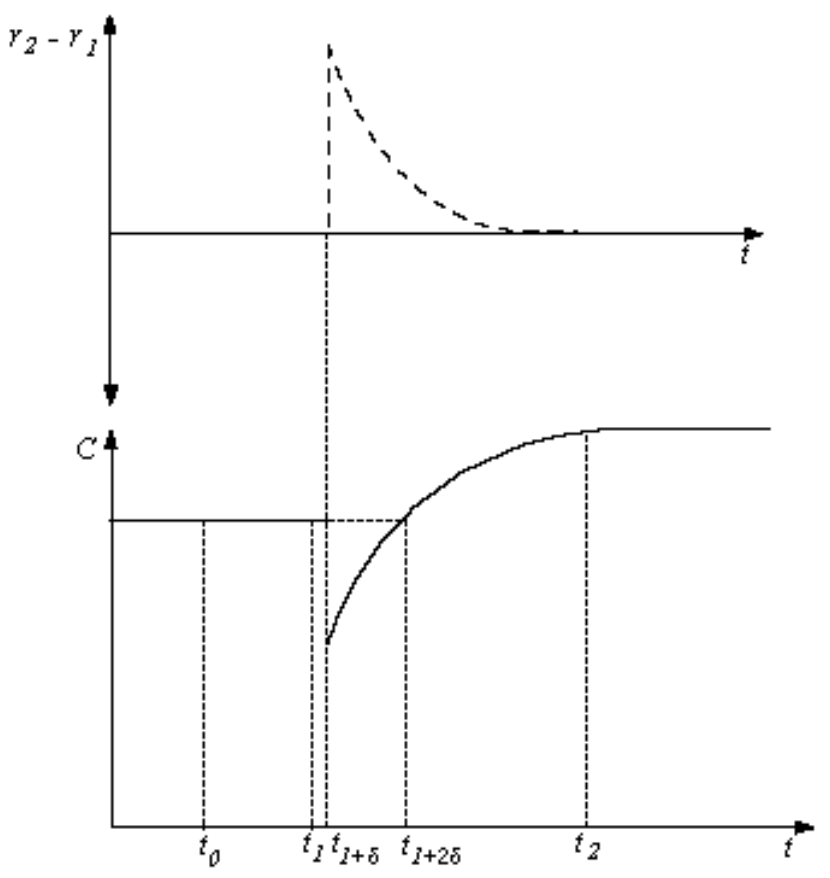

cial de inflación. Para ver esto más claramente, consideremos entonces la ecuación de Fisher: ${ }^{8}$

$$
i_{1}=r_{1}+E_{0} \pi_{1}
$$

siendo $\pi_{1}$ la inflación acumulada entre $t$ y $t+1$, y $r_{l}$ e $i_{l}$ las tasas de interés real y nominal, respectivamente, entre $t$ y $t+1$. Resolviendo para $r_{l}$ tenemos:

$$
r_{1}=i_{1}-E_{0} \pi_{1}
$$

${ }^{8}$ En la cual la tasa de interés nominal se ajusta en una relación 1 a 1 con la tasa de inflación esperada. Algunas versiones de la ecuación de Fisher han sido derivadas de modelos microfundamentados [véase, por ejemplo, Sargent (1987, cap. 6)]. La forma presentada en la ecuación (7) del texto principal supone que los elementos del lado derecho no exhiben ninguna convarianza entre sí. Pero, en ocasiones, la ecuación de Fisher se expresa como $i_{1}=r_{1}+E_{0} \pi_{1}+\operatorname{cov}\left(r, E_{0} \pi_{1}\right)$ lo cual plantea que la inflación esperada está determinada conjuntamente con los determinantes fundamentales de la tasa de interés real (Howitt, 1994). Sin embargo, otros autores (véase Summers, 1983) no han encontrado evidencia a favor de esa hipótesis. Sargent (1987, páginas 165-6) sugiere dos posibilidades en las que dicha covarianza puede ser nula. Primero, cuando no hay incertidumbre sobre la inflación futura y segundo, cuando los consumidores son neutrales al riesgo (véase también Arnwine, 2004). 
y suponiendo expectativas racionales:

$$
\pi_{1}=E_{0} \pi_{1}+\varepsilon_{1}
$$

siendo $\varepsilon_{1}$ una perturbación de valor esperado cero y varianza constante. Tomando (9) en cuenta, la expresión (8) puede ser re-escrita como:

$$
r_{1}=i_{1}-\pi_{1}+\varepsilon_{1}
$$

De forma similar para $r_{2}$ :

$$
r_{2}=i_{2}-\pi_{2}+\varepsilon_{2}
$$

Reemplazando (10) y (11) en (5) tenemos:

$$
\ln E_{t}\left[\frac{U^{\prime}\left(C_{t+1}\right)}{U^{\prime}\left(C_{t}\right)}\right]-\ln E_{t}\left[\frac{U^{\prime}\left(C_{t+2}\right)}{U^{\prime}\left(C_{t}\right)}\right]=\ln \beta+\left(i_{2}-i_{1}\right)-\left(\pi_{2}-\pi_{1}\right)+\mu_{2}
$$

siendo $\mu_{2}=\varepsilon_{2}-\varepsilon_{1}$. La ecuación (12) plantea una relación positiva entre el cambio esperado en la actividad económica y las tasas nominales de interés y negativa con el diferencial de inflación.

Estrella y Hardouvelis (1991) y Estrella y Mishkin (1996) sugieren la utilización de modelos probit para verificar la hipótesis de que la pendiente de la curva de rendimientos contiene información acerca de la trayectoria futura del crecimiento económico. Utilizando este enfoque, pero optando por un modelo logit ordenado en lugar de un probit (dado que el modelo de Arango y Melo (2005) que sirve de base para estimar el comportamiento de la actividad económica utiliza la función logística) nuestro modelo econométrico se convierte en:

$$
Q_{2,1}=F\left[\alpha_{0}+\alpha_{1}\left(i_{2}-i_{1}\right)_{t}-\alpha_{2}\left(\pi_{2}-\pi_{1}\right)\right]
$$

donde la variable dependiente es una medida de la probabilidad de un cambio de régimen de la actividad económica entre los períodos 1 y 2 . Es decir, que la economía pase de un buen momento económico a un momento difícil o al contrario. En lugar de utilizar el crecimiento del consumo, como lo señala nuestro modelo, utilizaremos el cambio de régimen del índice de producción industrial estimado con base en el modelo de Arango y Melo (2005). 


\section{Datos para Colombia}

La expresión (13) es la base de las estimaciones de este trabajo. Por lo tanto, se requieren series de expectativas de cambio en el régimen de actividad económica (auge o recesión), spread de tasas de interés y diferenciales de inflación.

En primer lugar, dado que en Colombia no hay indicador oficial del régimen en que se encuentra la economía, establecer si la economía está viviendo, en un momento determinado, condiciones de auge o recesión es algo que puede implicar juicios de valor. Por ello, tratando de ganar en objetividad para la identificación de los diferentes regímenes que han gobernado la economía colombiana, decidimos utilizar un modelo estadístico. La alternativa empleada es el modelo no lineal (LSTAR) estimado por Arango y Melo (2005), el cual propone la identificación de los regímenes a partir de la evolución del índice de producción real de la industria manufacturera. ${ }^{9}$ En el Anexo 1 a este trabajo se presentan los parámetros estimados del modelo.

En el Gráfico 1, aparece la función de transición estimada por Arango y Melo (2005). Allí el régimen superior, donde la función toma valores iguales a uno, identifica momentos buenos (de auge, expansión o aceleración) mientras que el régimen inferior, donde la función toma valores iguales a cero, identifica momentos difíciles (de recesión, depresión o desaceleración). Así, por ejemplo, entre diciembre de 1998 y noviembre de 1999, la función de transición estimada estuvo en el régimen inferior, lo cual sugiere un ambiente de recesión prolongada tal como aparece en los registros de algunos economistas en Colombia.

\section{GRAFICO 1
FUNCION DE TRANSICION EN EL TIEMPO}

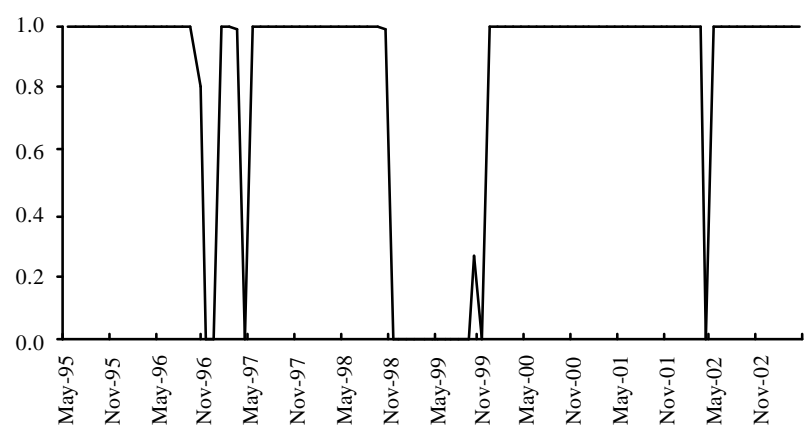

Fuente: Arango y Melo (2005)

\footnotetext{
${ }^{9}$ Existen muchas alternativas de escogencia de la variable asociada a la actividad económica como lo demuestran todos los estudios referenciados en la sección 2. Por ejemplo, para el caso de Colombia Arango y Flórez (2004) utilizan el cambio en el logaritmo del índice de producción industrial y el índice del valor de las ventas industriales. Este estudio muestra una evidencia clara del contenido informativo de la estructura a plazo real sobre las expectativas de la actividad económica futura. Una alternativa válida podría ser también una clasificación en tres regímenes, en lugar de dos, como la presentada por Johnson $(2001,2002)$ para el caso de Chile.
} 
La expresión (12) supone que se debe estimar el cambio esperado de actividad económica entre los momentos $t+1$ y $t+2$. Las diferentes posibilidades de cambio de régimen de la economía se resumen en el Cuadro 1, donde, como se dijo, cero refleja un período de recesión (depresión o desaceleración) y uno refleja un periodo de auge (expansión o aceleración) económico.

\section{CUADRO 1}

VALORES POSIBLES DE LA VARIABLE CORRESPONDIENTE AL CAMBIO DE REGIMEN

\begin{tabular}{cccc}
\hline \multirow{2}{*}{ En $(t+1)$} & Régimen & $\begin{array}{c}\text { Diferencia: } \\
(t+1)-(t+2)\end{array}$ & Equivalencia \\
\hline 0 & En $(t+2)$ & 0 & No hay cambio de régimen \\
& 1 & -1 & Cambio favorable \\
1 & 0 & 1 & Cambio adverso \\
& 1 & 0 & No hay cambio de régimen \\
\hline
\end{tabular}

La primera columna en el Cuadro 1 muestra los dos posibles estados de la economía en el momento $t+1$. A partir de allí, en el momento $t+2$ que se registra en la segunda columna, la economía puede tomar de nuevo uno de dos valores, bien sea que en el período anterior la economía haya estado en un buen momento o en un momento difícil. La tercera columna muestra la diferencia entre el valor de los estados que requiere la ecuación (12). Sobre esto, se deben tener en cuenta dos puntos. En primer lugar, el cambio en el régimen de actividad económica esperado toma un valor negativo, en lugar de uno positivo, como lo propone la ecuación (12), cuando se pasa de momentos económicos difíciles a momentos favorables. Esto sugiere alguna cautela al momento de analizar los signos de los coeficientes estimados en las secciones 5 y 6 . En segundo lugar, la definición de la variable dependiente hace equivalentes las situaciones en las que no hay cambio de régimen, bien sea porque se espera que la economía siga en un buen momento o porque se espera que siga en un momento difícil. ${ }^{10}$

Para calcular el spread de las tasas de interés, $i_{m}-i_{n}$, siendo $i_{m}$ e $i_{n}$ las tasas de interés nominal entre $t$ y $t+m$ y entre $t$ y $t+n$, respectivamente, se utilizó el promedio mensual del rendimiento nominal a 6, 12, 24 y 36 meses de los títulos $T E S$, tasa fija, emitidos por el gobierno nacional y transados en la Bolsa de Colombia y el Sistema Electrónico Nacional (SEN) para el periodo 1995:05 - 2003:06. Al final, el plazo de 36 meses fue eliminado debido a que la serie presentaba continuas interrupciones. ${ }^{11}$ Así, para $m$ se consideraron los plazos 12 y 24 mientras que para $n$ se tomaron los plazos 6 y 12 . Los spreads analizados fueron: $i_{24}-i_{12}, i_{24}-i_{6}$ y $i_{12}-i_{6}$ (Gráfico 2). Como sugiere la intuición económica y requiere el modelo econométrico, las series de spread de tasas de interés deben ser estacionarias,

\footnotetext{
${ }^{10}$ Sin embargo, esa equivalencia observacional es compatible con el modelo teórico.

${ }^{11}$ Interrupciones causadas por falta de transacciones.
} 
resultado que se verifica para las tres series, de acuerdo con las pruebas convencionales. En el Anexo 2 se consignan las pruebas sobre estacionaridad de las series.

GRAFICO 2

SPREAD DE TASAS DE INTERES

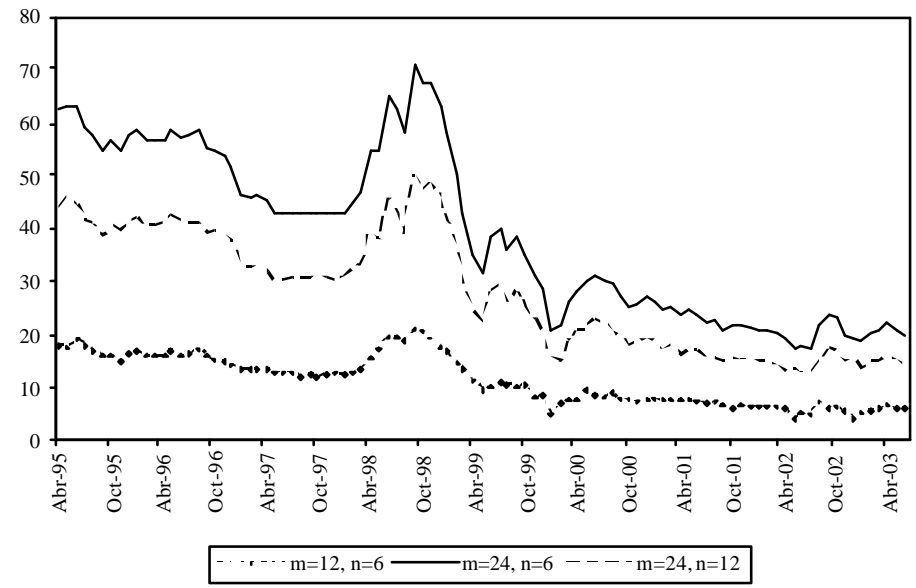

Los diferenciales de inflación para el periodo 1995:05 - 2003:06, $\pi_{m}-\pi_{n}$, se calcularon con base en cuatro definiciones diferentes ${ }^{12}$. La primera medida corresponde a la tasa de inflación total, calculada a partir del Índice de Precios al Consumidor (en adelante ITD). Las demás medidas corresponden a los tres indicadores de inflación básica calculados y utilizados actualmente por el Banco de la República: la inflación excluyendo el grupo de alimentos $(I B D)$, la inflación del núcleo inflacionario $20^{13}$ (IND) y la inflación del IPC excluyendo los alimentos primarios, los servicios públicos y los combustibles (ISCD). En el caso de $m$ se consideraron los plazos de 12 y 24 meses, mientras que para $n$ se tomaron los plazos 6 y 12 meses, de manera que los diferenciales de inflación considerados fueron: $\pi_{24}-\pi_{12}$, $\pi_{24}-\pi_{6}$ y $\pi_{12}-\pi_{6}$ (Gráfico 3 ).

Pruebas econométricas estándar $(A D F, K P S S)$ aplicadas a los diferenciales de inflación sugieren la existencia de raíces unitarias en todos los ellos con excepción de los correspondientes a 12-6 y 24-12 para ITD y 24-12 para IND(ver Anexo 2). Este resultado reduce las posibilidades empíricas de nuestro trabajo.

\footnotetext{
${ }^{12} \mathrm{La}$ inflación se calcula mediante la fórmula $\pi_{m}=\left[\left(I P C_{t+m} / I P C_{t}\right)-1\right]$. Por lo tanto, $\pi_{m} \mathrm{y} \pi_{n}$ corresponden a las tasas de inflación acumuladas entre $\mathrm{t}$ y $\mathrm{t}+\mathrm{m}$ y entre $\mathrm{t}$ y $\mathrm{t}+\mathrm{n}$, respectivamente. ${ }^{13}$ El núcleo inflacionario excluye del IPC total el $20 \%$ de la ponderación con aquellos ítemes que entre enero de 1990 y abril de 1999 registraron la mayor volatilidad de precios.
} 


\section{EVIDENCIA EMPÍRICA PARA COLOMBIA}

Dado que la variable dependiente puede tomar tres diferentes valores (1, 0 y -1 según el Cuadro 1), el modelo propuesto es un logit ordenado (acumulado) que estima explícitamente la probabilidad de que la variable dependiente tome el valor 1 (es decir, que la economía sufra un cambio adverso al pasar de un momento económico favorable a un momento difícil) o la probabilidad de que la variable dependiente tome valores 0 (es decir, la probabilidad acumulada de que sigamos en el mismo régimen -de auge o recesión-o que tengamos un cambio adverso de régimen económico), condicional a los valores del spread y del diferencial de inflación. ${ }^{14}$

\section{GRAFICO 3}

DIFERENCIAL DE INFLACIÓN A DISTINTOS PLAZOS

Inflación total $(I T D)$

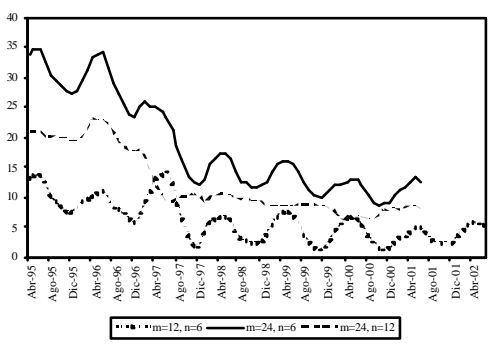

Inflación núcleo 20

$$
\text { (IND) }
$$

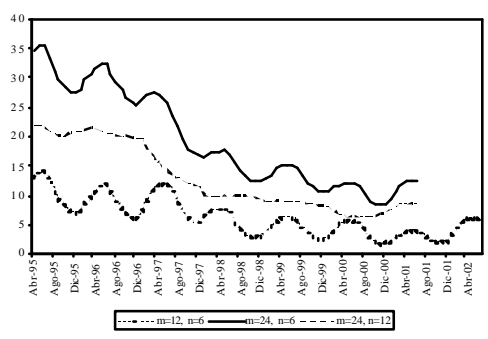

Inflación sin alimentos $(I B D)$

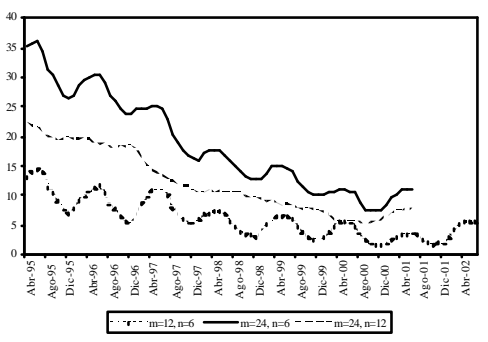

Inflación sin alimentos primarios, servicios públicos y combustibles $(I S C D)$

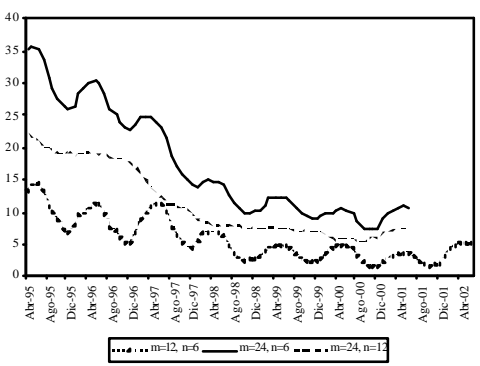

Para el proceso de estimación de la variable observable (que la actividad económica pase de un régimen a otro entre dos períodos o siga en el mismo régimen), considere una variable latente (no observable) $y_{i}^{*}$ equivalente a la probabilidad de cambiar de régimen o de seguir en el mismo, la cual depende linealmente de las variables explicatorias, $X$ :

\footnotetext{
${ }^{14}$ Note que la probabilidad de que la variable dependiente tome el valor $-1,[\mathrm{p}(-1)]$, es igual a 1 menos la probabilidad acumulada de que tome el valor $0,[1-\mathrm{p}(0)]$.
} 


$$
y_{i}^{*}=X_{i}^{\prime} \delta+\varepsilon_{i}
$$

\section{donde $\varepsilon_{i}$ es una variable al eatoria distribuidalogit. El valor observado de $y_{i}$ dependede $y_{i}^{*}$ de la siguiente manera:}

$$
y_{i}=\left\{\begin{array}{lc}
1 & \text { si } 0 \leq y_{i}^{*} \leq \gamma_{1} \\
0 & \text { si } \gamma_{1} \leq y_{i}^{*} \leq \gamma_{2} \\
-1 & \text { si } \gamma_{2} \leq y_{i}^{*} \leq \gamma_{3}
\end{array}\right.
$$

Las probabilidades de observar cada valor de $y_{i}$ están dadas por:

$$
\begin{aligned}
& \operatorname{Pr}\left(y_{i}=1 \mid X_{i}, \delta, \gamma\right)=F\left(\gamma_{1}-X_{i}^{\prime} \delta\right) \\
& \operatorname{Pr}\left(y_{i}=0 \mid X_{i}, \delta, \gamma\right)=F\left(\gamma_{2}-X_{i}^{\prime} \delta\right)-F\left(\gamma_{1}-X_{i}^{\prime} \delta\right) \\
& \operatorname{Pr}\left(y_{i}=-1 \mid X_{i}, \delta, \gamma\right)=F\left(\gamma_{3}-X_{i}^{\prime} \delta\right)-F\left(\gamma_{2}-X_{i}^{\prime} \delta\right)=1-F\left(\gamma_{3}-X_{i}^{\prime} \delta\right)
\end{aligned}
$$

siendo $F$ la función de distribución acumulada del error.

En el modelo logit ordenado del Cuadro 2, estimado por máxima verosimilitud, la variable dependiente toma los valores 1,0 y -1 . Los resultados muestran que los coeficientes estimados para predecir el cambio de régimen entre los próximos seis meses y el próximo año no son significativos (Cuadro 2$)^{15}$. Ello sugiere que la caída en el consumo y el aumento en la inversión, debido a un aumento en la tasa de interés, se compensan de manera que no se espera que la actividad económica agregada registre ninguna modificación estadísticamente significativa.

En general, los niveles de los coeficientes de este tipo de modelos son difíciles de interpretar. En su lugar, los autores suelen analizar el signo de los mismos (Allison, 1999). En ese sentido, es importante observar que el modelo tiene dos interceptos (dado que son tres los posibles valores de la variable dependiente). El primer intercepto que aparece en la Cuadro 2 (-1.8736) se interpreta como el logaritmo de la razón entre la probabilidad de que en el futuro suframos un cambio adverso en la actividad económica y su complemento (la probabilidad de que no haya cambio o de que el cambio sea favorable) cuando tanto el spread de tasas de interés como el diferencial de inflación son iguales a cero. El exponencial de ese número es 0.15 . Esto significa que la probabilidad de que en el futuro la economía

\footnotetext{
${ }^{15}$ Debe observarse, sin embargo, que el signo correspondiente al spread, es positivo lo cual estaría sugiriendo que en el futuro cercano, dado un aumento en la tasa de interés, se presentará una caída de la actividad económica. Esto es, el signo sugiere que la economía pasará de un momento favorable a uno difícil o seguirá en las mismas condiciones de crecimiento o recesión. Sin embargo, como se ha dicho, el coeficiente carece de significancia estadística.
} 
cambie hacia un estado desfavorable, con respecto a la probabilidad de que no cambie o de que cambie hacia uno favorable es de 0.15 a 1 . De igual manera, el segundo intercepto (2.1745) es el logaritmo de la razón entre la probabilidad de tener un cambio adverso en la economía o de no tener ningún cambio de régimen y su complemento. El exponencial de este número (8.79) nos dice que la probabilidad predicha de que los cambios de régimen tomen los valores 1 ó 0 es, casi, de 9 a 1. Considerando conjuntamente los valores anteriores, la mayor probabilidad de desempeño de la economía entre seis y doce meses adelante es que las cosas sigan como van.

\section{CUADRO 2}

MODELO LOGIT PARA ESTIMAR LA PROBABILIDAD DE QUE CAMBIE EL REGIMEN DE ACTIVIDAD ECONOMICA ENTRE 6 Y 12 MESES ADELANTE

\begin{tabular}{lrccc}
\hline & $\alpha$ & Error estándar & Estadística Z & Probabilidad \\
\hline Intercepto (1) & -1.8736 & 0.7858 & -2.3843 & 0.0171 \\
Intercepto (0) & 2.1745 & 0.8024 & 2.7099 & 0.0067 \\
Spread & 0.0123 & 0.0658 & 0.1869 & 0.8519 \\
ITD & -0.0488 & 0.0830 & -0.5879 & 0.5569 \\
\hline
\end{tabular}

En el Cuadro 3 aparecen los resultados para el cambio de régimen entre 12 y 24 meses adelante. En este caso se realiza la prueba sobre la irrelevancia de la forma como la variable dependiente se haga dicótoma, ${ }^{16}$ los efectos del spready del diferencial de inflación son siempre los mismos. Sin embargo, dicha hipótesis se rechaza de acuerdo con la prueba de proporcionalidad. Por tal razón, estimamos dos modelos logit: en el primero la variable dependiente toma valores 0 y -1 , mientras que en el segundo toma valores 1 y 0 . Los resultados aparecen en la parte inferior del Cuadro 3. Así, cuando la variable dependiente toma los valores de 0 y -1, el coeficiente correspondiente al spread toma un valor negativo mientras que cuando la variable dependiente toma valores 1 y 0 el coeficiente estimado para el spread, aunque no es significativo a niveles convencionales, toma un valor positivo. En suma, la evidencia sugiere que un incremento en el spread reduce la probabilidad de que se presente un cambio adverso en la economía.

\footnotetext{
${ }^{16}$ Es decir, que se pueden correr dos modelos logit convencionales en los que la variable dependiente tome los valores 0 y -1 ó 1 y 0 , respectivamente. El primero de estos logit se utilizaría para modelar la probabilidad de que haya un cambio favorable en la actividad económica y el segundo, la probabilidad de pasar a un momento difícil. La prueba de proporcionalidad verifica la hipótesis de que los coeficientes de ambos modelos logit son estadísticamente iguales. Esta prueba también se realizó en el caso anterior (cambio de régimen entre 6 y 12 meses adelante), sólo que allí la hipótesis no fue rechazada.
} 
CUADRO 3

MODELO LOGIT PARA ESTIMAR LA PROBABILIDAD DE QUE CAMBIE EL REGIMEN DE ACTIVIDAD ECONOMICA ENTRE 12 Y 24 MESES ADELANTE

\begin{tabular}{lcccc}
\hline & $\alpha$ & Error estándar & Estadística Z & Probabilidad \\
\hline Intercepto (1) & -0.1520 & 0.8237 & -0.1845 & 0.8536 \\
Intercepto (0) & 2.8397 & 0.8965 & 3.1675 & 0.0015 \\
Spread & -0.1046 & 0.0353 & -2.9631 & 0.0030 \\
ITD & 0.1531 & 0.0611 & 2.5057 & 0.0123 \\
Log Likelihood & -65.1108 & Test sobre supuesto de & 7.8322 \\
& & proporcionalidad & \\
P-seudo-R & & & & 0.0199 \\
& Variables & 0 y -1 de actividad económica & \\
Intercepto & 4.1068 & 1.4049 & 2.9232 & 0.0035 \\
Spread & -0.1627 & 0.0498 & -3.2671 & 0.0011 \\
ITD & 0.1917 & 0.0782 & 2.4514 & 0.0142 \\
& Variables 1 y & 0 de actividad económica & \\
Intercepto & -2.2556 & 1.1851 & -1.9033 & 0.0570 \\
Spread & 0.0653 & 0.0597 & 1.0938 & 0.2740 \\
ITD & -0.0707 & 0.0943 & -0.7497 & 0.4533 \\
& & & \\
\hline
\end{tabular}

Cuando el cambio de régimen analizado es el correspondiente a los meses 12 y 24 , pero en lugar de utilizar la inflación total (ITD) se utiliza la inflación núcleo $20(I N D)$, el modelo logit ordenado muestra que los coeficientes estimados son significativos, que los signos son los esperados y que el ajuste es altamente satisfactorio (Cuadro 4). De acuerdo con esto, un aumento en el spread de tasas de interés reduce la probabilidad de que entre un año y dos años adelante se pase de un buen ambiente económico a uno desfavorable. De igual manera, un mayor diferencial de inflación aumenta la probabilidad de pasar de un buen momento económico a un ambiente difícil.

En relación con el intercepto que resultó ser significativo, cuando el spread de las tasas de interés y el diferencial de inflación son iguales a cero, la probabilidad de que los cambios de régimen tomen valores entre 1 ó 0 es de 25.6 a 1 (=exp (3.2424)).

Los resultados de los ejercicios econométricos anteriores muestran que la estructura a plazo de las tasas de interés contiene información relevante para predecir la probabilidad de que se presenten cambios en el ambiente económico futuro. ${ }^{17}$ El mejor modelo logit ordenado para estimar dichos cambios se obtiene cuando se utiliza una de las definiciones de inflación básica utilizada por el Banco de la República, como es la inflación núcleo 20.

Estos resultados parecen sensatos de acuerdo con las predicciones del modelo teórico, en el sentido que el comportamiento de los signos se ajusta tanto

\footnotetext{
${ }^{17}$ Ninguno de los modelos presenta problemas de multicolinealidad.
} 
al modelo teórico como a la dinámica que de él se desprende: un aumento de las tasas de interés futuras no tiene impacto en la actividad económica en el futuro cercano pero sí en un plazo mayor.

CUADRO 4

MODELO LOGIT PARA ESTIMAR LA PROBABILIDAD DE QUE CAMBIE EL REGIMEN DE ACTIVIDAD ECONOMICA ENTRE 12 Y 24 MESES ADELANTE

\begin{tabular}{lcccc}
\hline & $\alpha$ & Error estándar & Estadística Z & Probabilidad \\
\hline Intercepto (1) & -0.1073 & 0.8733 & -0.1228 & 0.9022 \\
Intercepto (0) & 3.2424 & 0.9513 & 3.4083 & 0.0007 \\
Spread & -0.1617 & 0.0421 & -3.8409 & 0.0001 \\
IND & 0.2760 & 0.0718 & 3.8440 & 0.0001 \\
Log likelihood & -59.1743 & Test sobre supuesto de proporcionalidad & 2.2687 \\
P-seudo-R & 0.1566 & Valor-p del test de proporcionalidad & 0.3216 \\
\hline
\end{tabular}

\section{ENFOQUE (SOLAMENTE) EMPÍRICO: UTILIZACIÓN DE VARIABLES MONETARIAS}

El sentido del tipo de ejercicio, puramente empírico, que proponemos en esta sección es verificar la hipótesis de que, al incluir otras variables que no aparecen explícitamente en el modelo, el spread de tasas de interés no pierde contenido informativo sobre las expectativas de la evolución futura de la actividad económica, fundamentalmente en el plazo entre 12 y 24 meses adelante, que es cuando, según la evidencia ofrecida hasta ahora, se verifica satisfactoriamente dicha hipótesis.

La justificación a este ejercicio se encuentra únicamente en el interés que algunas veces pueden tener, no sólo para un banco central sino también para otros agentes del mercado, las investigaciones que consideran, además del spread de tasas de interés, otras variables económicas y financieras para predecir el comportamiento de la actividad económica futura. ${ }^{18}$

En este caso utilizamos el crecimiento de la base monetaria en términos nominales y reales, ya que fue la única variable de la que se obtuvo evidencia de regresión a la media ${ }^{19}$ (Anexo 2). Los resultados de estos ejercicios se presentan en los Cuadros 5 a $8 .{ }^{20}$

\footnotetext{
${ }^{18}$ Son muchos los estudios que emplean estrategias empíricas únicamente. Véanse, por ejemplo, Bernard y Gerlach (1996), Bosner-Neal y Morley (1997), Castellanos y Camero (2003), Estrella y Hardouvelis (1991) y Estrella y Mishkin (1995), entre muchos otros.

${ }^{19}$ Además del crecimiento en la base monetaria, también se hicieron pruebas con el crecimiento de $\mathrm{m} 1, \mathrm{~m} 2$ y $\mathrm{m} 3$.

${ }^{20}$ Los resultados presentados en los Cuadros 5 a 9 cumplen varios requisitos: que el coeficiente correspondiente al crecimiento del agregado monetario nominal o real sea significativo, que el valor- $p$ de la prueba de proporcionalidad supere el $12 \%$, y que las variables explicativas no exhiban alta colinealidad.
} 
Para el cambio de régimen entre 6 y 12 meses adelante (Cuadro 5) se presentan los resultados incluyendo el crecimiento contemporáneo (entre 6 meses y 12 meses adelante) de la base monetaria como variable adicional. Como se observa, el parámetro estimado para el crecimiento de la base monetaria resultó ser significativo, por lo que se puede señalar que esta variable contiene información sobre el ambiente económico que se espera vivir entre seis y doce meses adelante. El signo del coeficiente sugiere que un mayor valor del crecimiento contemporáneo de la base reduce la probabilidad de tener cambios de régimen adversos. Debe notarse que la inclusión del crecimiento de la base monetaria cambia el signo del coeficiente correspondiente al spread (ver Cuadro 2), aunque sigue sin ser significativo estadísticamente a este plazo; el diferencial de inflación, por su parte, es robusto en cuanto al signo del coeficiente pero, de nuevo, sigue sin significancia estadística.

CUADRO 5

MODELO LOGIT PARA ESTIMAR LA PROBABILIDAD DE QUE CAMBIE EL REGIMEN DE ACTIVIDAD ECONOMICA ENTRE 6 Y 12 MESES ADELANTE INCLUYENDO AGREGADOS MONETARIOS NOMINALES

\begin{tabular}{lcccc}
\hline & $\alpha$ & Estadística Z & Probabilidad & Indice LR (pseudo-R ${ }^{2}$ ) \\
\hline Intercepto (1) & -0.6712 & -0.7848 & 0.4326 & \\
Intercepto (0) & 4.1434 & 4.0820 & $<.0001$ & 0.1292 \\
Spread & -0.0224 & -0.3217 & 0.7476 & Valor-p del test de \\
ITD & -0.1094 & -1.2715 & 0.2035 & proporcionalidad \\
Ddbase & -0.1364 & -3.6681 & 0.0002 & 0.3532 \\
\hline
\end{tabular}

Cuando el agregado monetario se utiliza en términos reales, deflactado por el IPC, un mayor crecimiento monetario reduce la probabilidad de tener cambios adversos de la economía entre 6 y 12 meses adelante (Cuadro 6).

En síntesis, los resultados sugieren que el crecimiento de la base monetaria (nominal o real), contiene información que reduce la probabilidad de mostrar un cambio adverso en la economía en el futuro cercano.

Igual sucede con los cambios entre 12 y 24 meses adelante, cuando el diferencial de inflación se mide con la inflación núcleo (IND): tanto el crecimiento de la base monetaria nominal como real (Cuadros 7 y 8) contienen información sobre el desempeño económico futuro. En ambos casos el crecimiento monetario aumenta la probabilidad de que entre 12 y 24 meses se pase de un buen ambiente económico a uno desfavorable, contrario a lo que sucede con el spread de las tasas de interés, el cual reduce dicha probabilidad. Los estadísticos de estos modelos al igual que el ajuste muestran que se trata de representaciones adecuadas.

De acuerdo con los resultados de este enfoque empírico, el crecimiento de la base monetaria contiene información que ayuda a predecir la probabilidad del comportamiento futuro de la economía colombiana. Los resultados parecen más consistentes para cambios de régimen entre 12 y 24 meses adelante que entre 6 y 12 meses adelante, en el sentido que los signos de los coeficientes no se modifican 
al cambiar el crecimiento nominal por el real. Lo más importante es que las variables del modelo original siguen siendo significativas pese a la inclusión de la variable monetaria. Más aún, el spread siempre tiene un efecto que va con el modelo teórico para el plazo entre uno y dos años adelante.

CUADRO 6

MODELO LOGIT PARA ESTIMAR LA PROBABILIDAD DE QUE CAMBIE EL REGIMEN DE ACTIVIDAD ECONOMICA ENTRE 6 Y 12 MESES ADELANTE INCLUYENDO AGREGADOS MONETARIOS REALES

\begin{tabular}{lcccc}
\hline & $\alpha$ & Estadística Z & Probabilidad & Indice LR (pseudo- ${ }^{2}$ \\
\hline Intercepto (1) & -0.7679 & -0.9101 & 0.3628 & \\
Intercepto (0) & 3.9418 & 3.9815 & 0.0001 & 0.1127 \\
Spread & -0.0145 & -0.2115 & 0.8324 & Valor-p del test de \\
ITD & -0.2368 & -2.3620 & 0.0182 & proporcionalidad \\
RDdbase & -0.1377 & -3.4616 & 0.0005 & 0.3391 \\
\hline
\end{tabular}

CUADRO 7

MODELO LOGIT PARA ESTIMAR LA PROBABILIDAD DE QUE CAMBIE EL REGIMEN DE ACTIVIDAD ECONOMICA ENTRE 12 Y 24 MESES ADELANTE INCLUYENDO AGREGADOS MONETARIOS NOMINALES

\begin{tabular}{lrccc}
\hline & \multicolumn{1}{c}{$\alpha$} & Estadística Z & Probabilidad & Indice LR (pseudo- ${ }^{2}$ ) \\
\hline Intercepto (1 & -1.9127 & -0.1598 & 0.2461 & \\
Intercepto (0) & 1.5327 & 0.9460 & 0.3441 & 0.2376 \\
Spread & -0.1936 & -3.6696 & 0.0002 & Valor-p del test de \\
IND & 0.4084 & 4.1840 & $>.00001$ & proporcionalidad \\
Ddbase (12) & 0.0832 & 2.4517 & 0.0142 & 0.6824 \\
\hline
\end{tabular}

CUADRO 8

MODELO LOGIT PARA ESTIMAR LA PROBABILIDAD DE QUE CAMBIE EL REGIMEN DE ACTIVIDAD ECONOMICA ENTRE 12 Y 24 MESES ADELANTE INCLUYENDO AGREGADOS MONETARIOS REALES

\begin{tabular}{lcccc}
\hline & $\alpha$ & Estadística Z & Probabilidad & Indice LR (pseudo-R ${ }^{2}$ ) \\
\hline Intercepto (1) & -2.9765 & -1.7028 & 0.0886 & \\
Intercepto (0) & 0.6411 & 0.3820 & 0.7024 & 0.2693 \\
Spread & -0.1847 & -3.4536 & 0.0006 & Valor-p del test de \\
IND & 0.5134 & 4.3847 & $<.00001$ & proporcionalidad \\
RDdbase(12) & 0.1373 & 3.0318 & 0.0024 & 0.4784 \\
\hline
\end{tabular}




\section{Conclusiones}

En este trabajo se verifica la hipótesis de que la estructura a plazo de tasas de interés contiene información sobre el desempeño de la actividad económica futura en Colombia. La información sobre las tasas de interés ha sido recientemente producida en el mercado colombiano de títulos de deuda de renta fija.

Debido a la carencia de una variable "oficial" sobre el régimen que vive la actividad económica (depresión, aceleración, auge, etc.) se utiliza como variable indicativa del desempeño de la economía la estimada por Arango y Melo (2005), quienes, por medio de un modelo no lineal tipo LSTAR, identifican los distintos regímenes que atraviesa la economía colombiana de acuerdo con el índice de producción industrial.

En tal sentido, la economía puede pasar, en el futuro, de un momento bueno a uno malo, de uno malo a uno bueno, o puede continuar igual (bien o mal). La probabilidad de la existencia de cualquiera de estos tres tipos de cambios de régimen es condicional al spread de tasas de interés y al diferencial de inflación entre 6 y 12 meses y entre 12 y 24 meses adelante por medio de un modelo multilogit.

Con esta metodología se obtiene evidencia en favor de la hipótesis de que la estructura a plazo de las tasas de interés contiene información sobre el desempeño futuro de la actividad económica.

Los resultados sugieren que para el período más cercano (entre 6 y 12 meses adelante), un aumento en el spread de tasas de interés no predice un cambio particular de régimen dada la falta de significancia del coeficiente asociado al spread de tasas de interés; durante este período el aumento de la inversión se puede compensar con la caída en el consumo de manera que el efecto neto en el producto no es de una magnitud importante, lo cual se ajusta a la dinámica que sugiere el modelo teórico de un agente representativo.

Cuando los cambios de régimen analizados cubren el período de 12 y 24 meses adelante, los signos de los coeficientes estimados indican que un aumento en el spread de las tasas de interés reduce la probabilidad de pasar de buenos momentos a momentos difíciles. Esto es justamente lo que predice el modelo teórico.

Para los cambios de régimen esperados entre 12 y 24 meses adelante, el mejor modelo logit ordenado se obtiene cuando se emplea una de las definiciones de inflación básica utilizada por el Banco de la República como es la inflación núcleo 20 (IND), la cual se genera excluyendo del IPC total el 20\% de la ponderación con aquellos ítemes que entre enero de 1990 y abril de 1999 registraron la mayor volatilidad de precios.

Finalmente, la inclusión de variables monetarias de manera ad hoc no hace perder capacidad informativa al spread de tasas de interés sobre el ambiente económico entre 12 y 24 meses adelante, aunque las variables monetarias también tienen capacidad predictiva. 


\section{REFERENCIAS}

Allison, P.D. (1999), Logistic Regression Using the SAS System. Theory and Application, SAS Institute Inc. Books by users, Cary.

Arosemena, A.M. y L.E. Arango (2002), "Lecturas Alternativas de la Estructura a Plazo: una Breve Revisión de la Literatura,” Borradores de Economía, 223, Banco de la República.

Arango, L.E y L.A. Flórez (2004), "Expectativas de Actividad Económica en Colombia y Estructura a Plazo: un Poco más de Evidencia," Ensayos sobre Política Económica, 46.

Arango, L.E. y L.F. Melo (2005), "Expansions and Contractions in Brazil, Colombia and Mexico: a View Through Non-linear Models," Journal of Development Economics, próximo a aparecer.

Arnwine, A. (2004), The Fisher Equation and Output Growth, Working Paper, Bilken University.

Bernard, H. y S. Gerlach (1996), "Does the Term Structure Predict Recessions?: the International Evidence," BIS Working Papers, 37.

Bosner-Neal, C. y T. Morley (1997), "Does the Yield Spread Predict Real Economic Activity? A Multicountry Analysis," Federal Reserve Bank of Kansas City Economic Review, Third quarter, 37-53.

Castellanos, S. y E. Camero (2003), "La Estructura Temporal de Tasas de Interés en México: ¿Puede ésta Predecir la Actividad Económica Futura?’” Revista de Análisis Económico, 18 (2): 33-66.

Dotsey, M. (1998), “The Predictive Content of the Interest Rate Term Spread for Future Economic Growth," Federal Reserve Bank of Richmond Economic Quarterly, (Spring) 84 (3): 31-51.

Estrella, A. y G. Hardouvelis (1991), "The Term Structure as a Predictor of Real Economic Activity," Journal of Finance, 46 (2): 555-576.

Estrella, A. y F. Mishkin (1995), "Predicting U.S. Recessions: Financial Variables as Leading Indicators," NBER Working Paper Series, 5379.

Estrella, A. y F. Mishkin (1996), "The Yield Curve as a Predictor of U.S. Recessions, Current Issues in Economics and Finance," Federal Reserve Bank of New York, 1- 6.

Fama, E.F. (1986), “Term Premiums and Default Premiums in Money Markets,” Journal of Financial Economics, 17: 75-198.

Fisher, I . (1097), The Rate of Interest, New York: MacMillan.

Granger, C. W. J. y T. Teräsvirta (1993), Modelling nonlinear economic relationships, Oxford University Press, Oxford.

Harvey, C.R. (1988), “The Real Term Structure and Consumption Growth," Journal of Financial Economics, 22: 305-333.

Harvey, C.R. (1997), "The Relation Between the Term Structure of Interest Rates and Canadian Economy Growth," Canadian Journal of Economics, XXX, 1: 169-93.

Howitt, P. (1994), Fisher Effect, New Palgrave Dictionary of Money \& Finance, London: Macmillan Press. 
Hu, Z. (1993), “The Yield Curve and Real Activity,” IMF Staff papers, 40, 4: 781-806.

Johnson, C. A. (2001), "Un Modelo de Switching para el Crecimiento en Chile," Cuadernos de Economía, 38 (115): 291-319.

Johnson, C. A. (2002), "Una Aplicación del Modelo de Cambio de Régimen para el Crecimiento y Evolución del Tipo de Cambio Nominal en Chile," El Trimestre Económico, 273: 65-94.

Kim, A. K. y P. Limpaphayom (1997), “The Effect of Economic Regimes on the Relation Between Term Structure and Real Activity in Japan,"Journal of Economics and Business, 49: 379-92.

Maurer, M.; M. C. Uribe y J. Birchenal (1996), "El Sistema de Indicadores Líderes para Colombia," Archivos de Macroeconomía, 49. DNP.

Melo, A.; M. French y N. Langebaek (1988), "El Ciclo de Referencia de la Economía Colombiana," Ministerio de Hacienda, 43-61.

Melo, L. F.; F. H. Nieto y M. Ramos (2003), “A Leading Index for the Colombian Economic Activity,” Borradores de Economía, 243, Banco de la República.

Sargent, T.J. (1987), “Dynamic Macroeconomic Theory,” London: Harvard University Press.

Summers, L. (1984 ), “The Nonadjustment of Nominal Interest Rates: a Study of the Fisher Effect," in Macreconomics, Prices and, Quantities: Essays in Memory of Arthur Okun, ed. J. Tobin, Washington, DC: The Brooking Institution.

Teräsvirta, T. (1998), "Modelling Economic Relationships with Smooth Transition Regressions," in Handbook of Applied Economic Statistics, A. Ullah and D.E.A. Giles (Eds.), Marcel Dekker, New York. 
ANEXO 1

MODELO LSTAR PARA EL INDICE DE PRODUCCION REAL (MMM) DE COLOMBIA

\begin{tabular}{lcccc}
\hline & Coeficiente & $\begin{array}{c}\text { Error standard } \\
\text { parte lineal }\end{array}$ & $t$-valor & Probabilidad \\
\hline Constant & -0.034 & 0.012 & -2.738 & 0.006 \\
$y_{t-1}$ & -0.516 & 0.060 & -8.517 & $<.00001$ \\
$y_{t-7}$ & -1.221 & 0.319 & -3.830 & $<.00001$ \\
$y_{t-10}$ & -0.157 & 0.053 & -2.991 & 0.003 \\
$y_{t-12}$ & -1.416 & 0.327 & -4.326 & $<.00001$ \\
& Parte no lineal (Variable de transición: $\left.\Delta_{12} y_{t-1}\right)$ & \\
Constant & 0.037 & 0.012 & 2.960 & 0.003 \\
$\hat{\gamma}$ & 69.501 & 93.210 & 0.745 & 0.456 \\
$\hat{c}$ & -0.0772 & 0.003 & -24.519 & $<.00001$ \\
$y_{t-2}$ & -0.201 & 0.065 & -3.069 & 0.002 \\
$y_{t-7}$ & 1.175 & 0.323 & 3.637 & $<.00001$ \\
$y_{t-12}$ & 1.484 & 0.333 & 4.445 & $<.00001$ \\
\hline
\end{tabular}

Fuente: Arango y Melo (2005). 


\begin{tabular}{|c|c|c|c|}
\hline & \multicolumn{2}{|c|}{$A D F$} & \multirow[t]{2}{*}{ KPSS } \\
\hline & Estadística $t$ & $\begin{array}{c}\text { Nivel de } \\
\text { significancia } Q\end{array}$ & \\
\hline \multicolumn{4}{|l|}{ Diferencia 12_6 } \\
\hline ITD & $-3.4856 *$ & 0.2047 & $0.1408 *$ \\
\hline IBD & -2.0885 & 0.9367 & 0.1591 \\
\hline IND & -2.5314 & 0.9080 & 0.1584 \\
\hline ISCD & -1.6446 & 0.5109 & 0.1991 \\
\hline Spread & $-3.1156 *$ & 0.5109 & $0.0749 *$ \\
\hline \multicolumn{4}{|l|}{ Diferencia 24_12 } \\
\hline ITD & -2.5527 & 0.7304 & $0.1675 *$ \\
\hline IBD & -1.7529 & 0.0069 & 0.1769 \\
\hline IND & $-3.4165 *$ & 0.2339 & $0.0747 *$ \\
\hline ISCD & -2.0980 & 0.0057 & 0.2053 \\
\hline Spread & $-3.2752 *$ & 0.3257 & $0.0769 *$ \\
\hline \multicolumn{4}{|l|}{ Diferencia 24_6 } \\
\hline ITD & -1.6278 & 0.0003 & 0.1967 \\
\hline IBD & -2.5215 & 0.0005 & 0.1987 \\
\hline IND & -3.1469 & 0.0000 & 0.1845 \\
\hline ISCD & -2.1309 & 0.5109 & 0.2194 \\
\hline Spread & $-3.5532 *$ & 0.9677 & $0.0763 *$ \\
\hline \multicolumn{4}{|l|}{ Agregados monetarios } \\
\hline Base 12_6 nominal & -3.1345 & 0.6450 & 0.0507 \\
\hline Base 24_12 nominal & -3.5644 & 0.5065 & 0.0909 \\
\hline Base $12 \_6$ real & -3.2820 & 0.8722 & 0.0502 \\
\hline Base 24_12 real & $-3.6863 *$ & 0.5509 & 0.0779 \\
\hline
\end{tabular}

Valores críticos ADF: $5 \%=-3.4557 ; 10 \%=-3.159$; KPSS: $5 \%=0.146 ; 2.5 \%=0.176$ (rezago 8). 